\title{
Immunohistochemical markers in diagnosis of papillary thyroid carcinoma: utility of HBME1 combined with CK19 immunostaining
}

\author{
Michel R Nasr ${ }^{1}$, Sanjay Mukhopadhyay ${ }^{2}$, Shengle Zhang ${ }^{1}$ and Anna-Luise A Katzenstein ${ }^{1}$ \\ ${ }^{1}$ Department of Pathology, State University of New York Upstate Medical University, Syracuse, NY, USA and \\ ${ }^{2}$ Department of Pathology, Mayo Clinic College of Medicine, Rochester, MN, USA
}

\begin{abstract}
Papillary thyroid carcinoma and its variants can be difficult to distinguish from cellular adenomatous nodules. Prior studies have advocated various antibodies to aid in the differential diagnosis, but there is little agreement on their utility. We undertook this study to evaluate immunohistochemical markers in the diagnosis and differential diagnosis of papillary thyroid carcinoma. Ten cases of papillary thyroid carcinoma were initially stained for HBME1, CK19, fibronectin1, Ki-67, Calretinin, p16, SFTPB and CITED1. Additionally, two previously untested antibodies to molecules that have been found to be upregulated in papillary thyroid carcinoma (CST6 and EPS8) were also evaluated. Of these, only HBME1, CK19 and fibronectin1 showed diagnostic utility. These three markers were then further evaluated in 51 papillary thyroid carcinomas and 57 benign thyroids. HBME1 was the most sensitive and specific marker, staining 49/51 papillary thyroid carcinomas and only $4 / 57$ benign thyroids. CK19 was equally sensitive staining all 51 papillary thyroid carcinomas, but it was nonspecific staining 39 of 57 benign thyroids. A negative result, however, was helpful in excluding papillary thyroid carcinoma. Fibronectin 1 was positive in $35 / 51$ papillary thyroid carcinomas $(69 \%)$ and $4 / 57(7 \%)$ benign thyroids, but its utility was hampered by high background staining. These findings suggest that the combination of HBME1 and CK19 has the greatest diagnostic utility in the differentiation of papillary thyroid carcinoma from its benign mimics.
\end{abstract}

Modern Pathology (2006) 19, 1631-1637. doi:10.1038/modpathol.3800705; published online 22 September 2006

Keywords: papillary thyroid carcinoma; HBME1; CK19; Fibronectin1

Carcinoma of the thyroid is the most common endocrine malignancy with approximately 19500 new cases diagnosed each year in the United States. ${ }^{1}$ Papillary thyroid carcinoma constitutes about $80 \%$ of all thyroid malignancies. ${ }^{2}$ The follicular variant is the most common subtype. ${ }^{3-5}$ The diagnosis of papillary thyroid carcinoma is based on architectural features combined with nuclear clearing, overlapping, grooves and pseudoinclusions. In the absence of papillary architecture, accurate differentiation of the follicular variant of papillary thyroid carcinoma from cellular adenomatous nodules may be challenging. Less commonly, papillary hyperplastic nodules may be difficult to distinguish from papillary thyroid carcinoma. Several immunostains have been proposed to aid in the diagnosis of papillary thyroid carcinoma, including CK19, ${ }^{6-9}$ HBME1, ${ }^{6,10-12}$ FN1 (Fibronectin1), ${ }^{13,14}$ CITED1

Correspondence: Dr MR Nasr, MD, Department of Pathology, State University of New York Upstate Medical University, 750 East Adams Street, Syracuse, NY 13210, USA.

E-mail: nasrm@upstate.edu

Received 28 July 2006; revised and accepted 24 August 2006; published online 22 September 2006
(Cbp/p300 Interacting Transactivators with glutamic acid [E] and aspartic acid [D]-rich C-terminal domains, also known as melanocyte-specific gene 1) and SFTPB (Surfactant, pulmonary-associated Protein B). ${ }^{15,16}$ Although galectin-3 was initially shown to have utility in the differential diagnosis between benign and malignant thyroid lesions, ${ }^{17,18}$ recent studies suggest that it is not reliable. ${ }^{19-21}$ Several other novel markers have been identified as a result of molecular analysis of papillary thyroid carcinoma, including CST6 (Cystatin E/M) and EPS8 (Epidermal growth factor receptor kinase Substrate) genes, ${ }^{15}$ but these have not been tested immunohistochemically. We undertook this study to reevaluate established antibodies and to test the utility of novel markers that have not yet been assessed in the diagnosis of papillary thyroid carcinoma.

\section{Materials and methods}

Tissue Samples

One hundred and eight thyroidectomy specimens were selected from the files of the Department of 
Pathology, University Hospital, Syracuse, NY between 1999 and 2005. These consisted of 51 papillary thyroid carcinomas (including 20 classic papillary thyroid carcinomas, 10 follicular variants and 21 papillary microcarcinomas) and 57 benign thyroid lesions (including 10 normal thyroids, 10 cellular adenomatous nodules, eight Graves disease, four papillary hyperplastic nodules, six follicular adenomas and 19 cases of Hashimoto's thyroiditis). All cases had hematoxylin \& eosin (H\&E) stains available for review and paraffin blocks for immunohistochemical staining. The H\&E slides were reviewed by all authors independently and the diagnosis were agreed upon using well-established histopathologic criteria. All authors independently reviewed the H\&E-stained sections and interpreted immunohistochemical staining results. Any disagreement in the histopathologic diagnosis or differences in interpreting the immunostaining results were resolved by consensus. Ten cases of papillary thyroid carcinoma were initially stained with HBME1, CK19, FN1, Ki-67, Calretinin, p16, SFTPB, CITED1, CST6 and EPS8. Of these, the most useful and discriminatory markers were HBME1, CK19 and FN1. Only these three markers were then further evaluated in all cases $(n=108)$.

\section{Immunohistochemistry}

Immunohistochemistry was performed on formalinfixed, paraffin-embedded (4- $\mu \mathrm{m}$ thick) sections using a standard technique (streptavidin-biotinperoxidase technique) with appropriate positive and negative controls. The following panel of antibodies was used: HBME1 (Dako, M3505, 1:50), CK19 (Neomarkers, Ks19.1, 1:50), FN1 (Dako, A0245, 1:2000), Ki-67 (Biogenex, Ki88, 1:40), CITED1 (Neomarkers, RP-9219-P, 1:50), SFTPB (Neomarkers, ES-CD3, 1:30), Calretinin (Zymed, PAD:DC8, 1:50), p16 (Neomarkers, 16P07, 1:100) CST6 (Abcam, AB11201, 1:10) and EPS8 (Abgent, AP8148a, 1:10). On appropriate negative controls, primary antibody was substituted with Tris-buffered saline. For all antibodies, immunoreactivity was considered positive if $>10 \%$ of follicular epithelial cells stained. ${ }^{13}$ The following staining patterns were considered positive: for HBME1, membrane staining along lateral and abluminal surfaces; ${ }^{13}$ for Ki-67 and p16, nuclear expression and for CK19, FN1, CITED1, Calretinin, SFTPB, CST6 and EPS8, cytoplasmic expression.

\section{Results}

The results of immunohistochemical staining in papillary thyroid carcinoma are summarized in Table 1. Forty-nine out of 51 (96\%) papillary thyroid carcinomas were positive for HBME1 (Figures 1-3) whereas the adjacent normal thyroid tissue was consistently negative. Only membranous (basolateral) staining was considered positive. Although cytoplasmic or luminal membrane staining was observed in many cases, these patterns of staining without basolateral membrane staining were not considered positive. Membrane staining with HBME1 was strong, with a cleanly negative background.

Table 2 shows the results of immunohistochemical staining in benign thyroid lesions. HBME1 stained only $4 / 57$ (7\%); positive staining was only observed in Hashimoto's thyroiditis (four of 19 cases). These cases showed strong membrane staining for HBME1 in small foci of non-Hürthle epithelial cells. In three of these four cases, the HBME1-positive epithelial foci showed some nuclear features of papillary thyroid carcinoma such as clearing, occasional grooves and slight overlapping, but these changes were not sufficiently well developed to allow a clear-cut morphologic diagnosis of papillary thyroid carcinoma. In the fourth case, nuclear features of papillary thyroid carcinoma were not identified in the HBME1-positive areas.

Cytoplasmic HBME1 staining in the Hürthle cells without membrane staining was commonly

Table 1 Immunohistochemical expression of HBME1, FN1, CK19, CITED1, SFTPB, Calretinin, p16, Ki-67, CST6 and EPS8 in papillary thyroid carcinoma subtypes

Positive cases/total cases assessed (\%)

\begin{tabular}{|c|c|c|c|c|}
\hline & $\begin{array}{c}\text { Classic papillary thyroid } \\
\text { carcinoma }\end{array}$ & $\begin{array}{c}\text { Follicular variant of papillary } \\
\text { thyroid carcinoma }\end{array}$ & $\begin{array}{c}\text { Papillary } \\
\text { microcarcinoma }\end{array}$ & $\begin{array}{l}\text { Papillary thyroid } \\
\text { carcinoma (total) }\end{array}$ \\
\hline HBME1 & $19 / 20(95)$ & $9 / 10(90)$ & $21 / 21(100)$ & 49/51 (96) \\
\hline CK19 & $20 / 20(100)$ & $10 / 10(100)$ & $21 / 21(100)$ & $51 / 51(100)$ \\
\hline FN1 & $20 / 20(100)$ & $7 / 10(70)$ & $8 / 21(38)$ & $35 / 51(69)$ \\
\hline CITED1 & $19 / 19(100)$ & $7 / 7$ (100) & NA & $26 / 26(100)$ \\
\hline SFTPB & $5 / 19(26)$ & $0 / 7(0)$ & NA & $5 / 26(19)$ \\
\hline Ki-67 & $18 / 19(95)$ & $0 / 7(0)$ & NA & $18 / 26(62)$ \\
\hline Calretinin & $3 / 10(30)$ & NA & NA & $3 / 10(30)$ \\
\hline p16 & $10 / 10(100)$ & NA & NA & $10 / 10(100)$ \\
\hline CST6 & $8 / 10(80)$ & NA & NA & $8 / 10(80)$ \\
\hline EPS8 & $10 / 10(100)$ & NA & NA & $10 / 10(100)$ \\
\hline
\end{tabular}



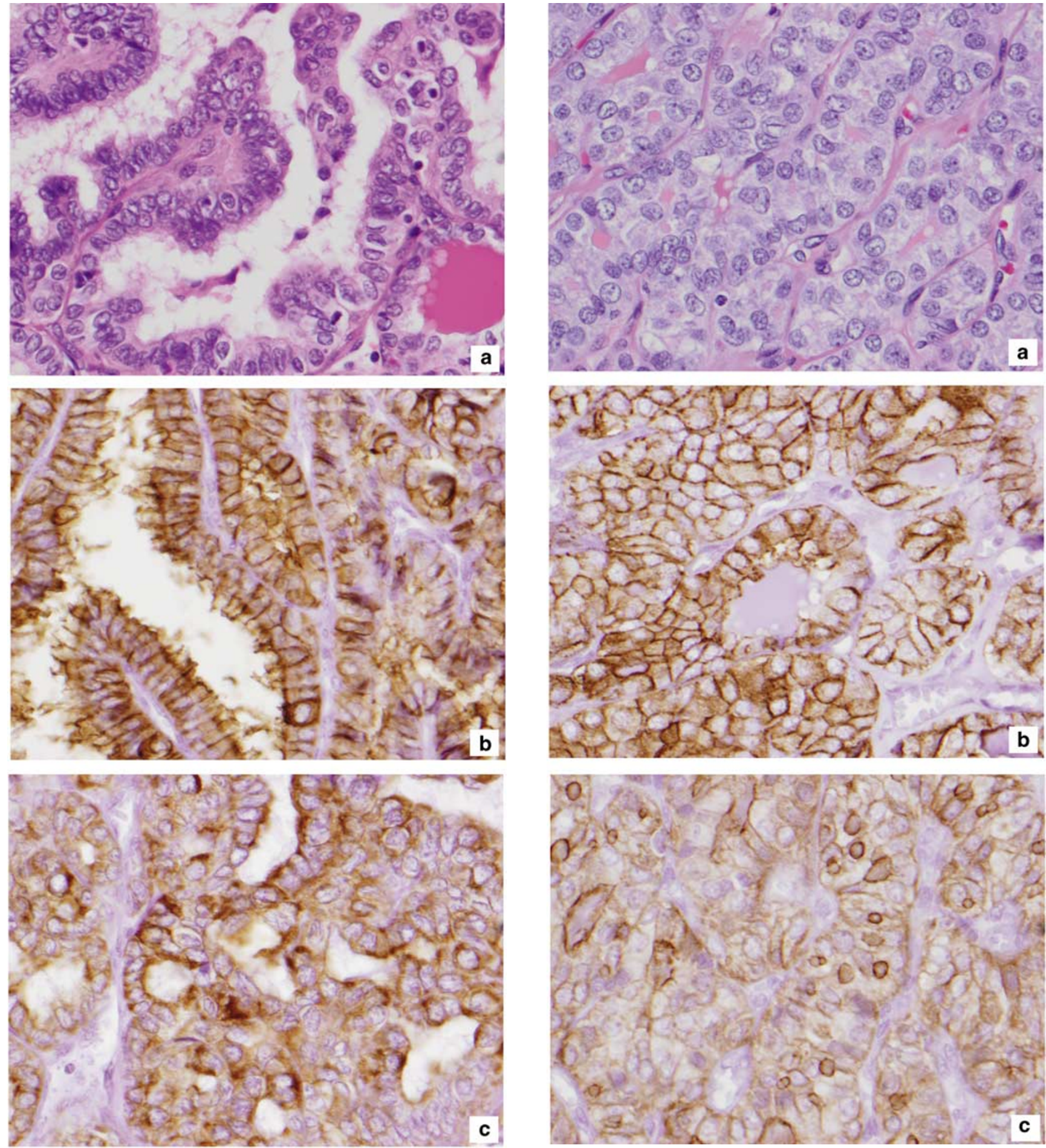

Figure 1 Papillary thyroid carcinoma, classic. (a) Hematoxylineosin $(\mathrm{H} \& \mathrm{E})(\times 100)$. (b) Strong membrane staining for HBME1 $(\times 100)$. (c) Cytoplasmic and membrane positivity for CK19 $(\times 100)$.

Figure 2 Follicular variant of papillary thyroid carcinoma. (a) $\mathrm{H} \& \mathrm{E}(\times 100)$. (b) Membrane positivity for HBME1 $(\times 100)$ (c) Positive staining for CK19 $(\times 100)$.

observed, but this staining pattern was considered negative. Overall, HBME1 was $96 \%$ sensitive and $93 \%$ specific for papillary thyroid carcinoma.

CK19 was positive in all $51(100 \%)$ papillary thyroid carcinomas (Figures 1-3). The staining

pattern was predominantly cytoplasmic with frequent enhancement at the cell membrane, and reactivity was strong and diffuse in most cases. Of the 57 benign lesions, 39 (68\%) were CK19 positive, including all 10 normal thyroids, 5/10 adenomatous 

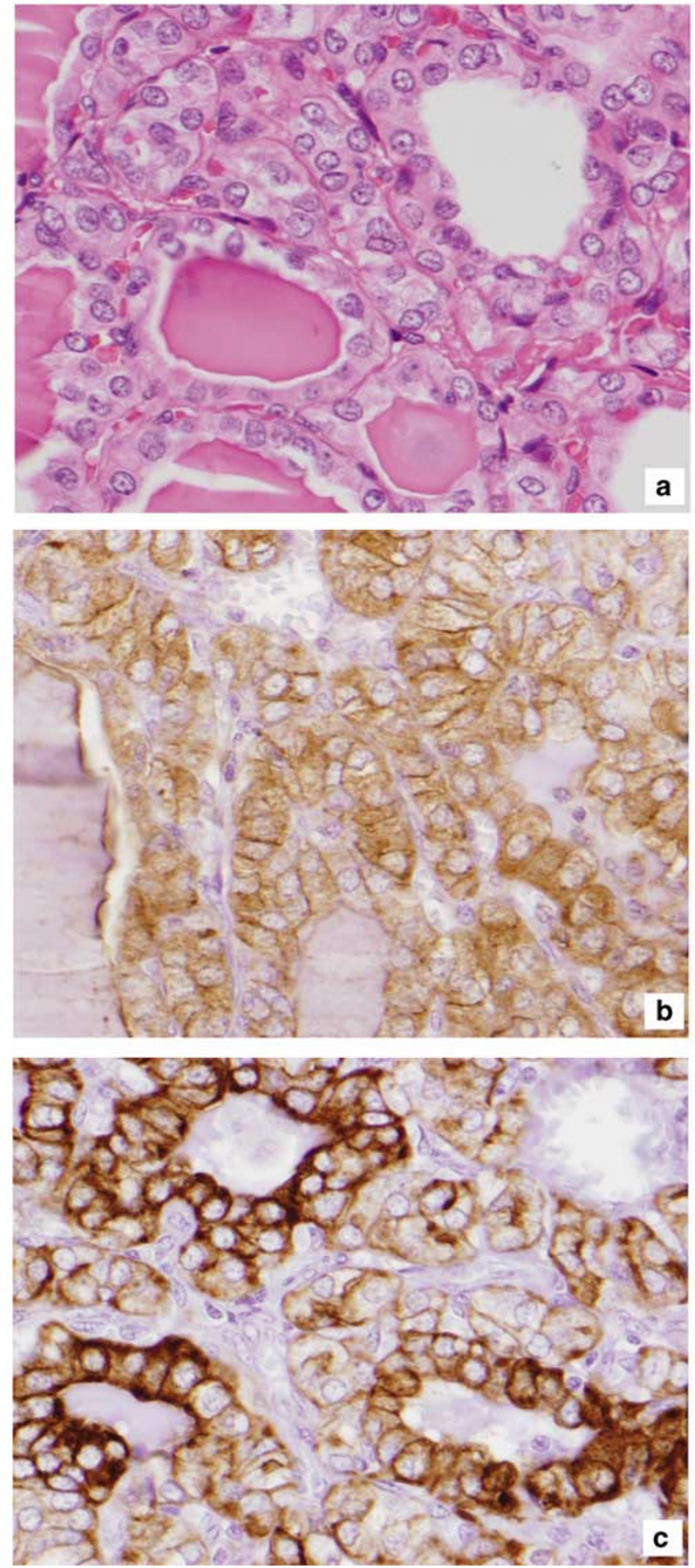

Figure 3 Papillary microcarcinoma. (a) H\&E $(\times 100)$. (b) Tumor cells are positive for HBME1 $(\times 100)$. (c) Strong cytoplasmic positivity for CK19 ( $\times 100)$.

nodules (Figure 4), 2/8 Graves, 1/4 papillary hyperplastic nodules, 5/6 follicular adenomas and 16/19 Hashimoto's thyroiditis. Although one of four papillary hyperplastic nodules was positive, only $15 \%$ of cells stained. Staining intensity was weak and diffuse in most of these benign lesions and the

staining pattern was predominantly cytoplasmic with frequent membrane enhancement. The sensitivity of CK19 for papillary thyroid carcinoma was $100 \%$ but the specificity was only $32 \%$.

FN1 positivity was seen in $35 / 51$ papillary thyroid carcinomas $(69 \%)$ and $4 / 57(7 \%)$ benign thyroid lesions, resulting in a sensitivity and specificity of 96 and $93 \%$, respectively. Immunoreactivity for FN1 was mostly focal, weak and cytoplasmic. Significant background staining was present in all cases and made interpretation difficult. None of the eight Graves' disease cases, four papillary hyperplastic nodules or 10 normal thyroids expressed FN1. However, FN1 positivity was seen in a few benign lesions, including 1/10 adenomatous nodule, 2/6 follicular adenomas and 1/19 Hashimoto's thyroiditis.

Thirty-five of 51 (69\%) papillary thyroid carcinomas were positive for all three stains (HBME1, CK19 and FN1). Classical papillary thyroid carcinomas had a higher rate of positivity (96\% HBME1, $100 \%$ CK19, 100\% FN1) than the follicular variant (90\% HBME1, 100\% CK19, 70\% FN1). All cases of papillary microcarcinoma were positive for HBME1 and CK19, but FN1 was positive in only $38 \%$ of cases.

Cytoplasmic CITED1 expression was seen in all 26 papillary thyroid carcinomas and in 30/31 benign thyroid lesions. SFTPB staining was generally weak and focal (5/26 papillary thyroid carcinomas and 1/31 benign thyroids). Nuclear expression of Ki-67 was very rare but cytoplasmic Ki-67 expression was present in 18/26 papillary thyroid carcinomas and 4/32 nonneoplastic thyroid lesions. Calretinin was expressed in $3 / 10$ papillary thyroid carcinomas but none of 10 benign lesions. EPS8 and p16 showed $100 \%(10 / 10)$ positivity in papillary thyroid carcinomas and $80 \%(8 / 10)$ in benign lesions. CST6 stained $80 \%(8 / 10)$ papillary thyroid carcinomas and $73 \%(11 / 15)$ benign thyroid lesions.

\section{Discussion}

Differentiating the follicular variant of papillary thyroid carcinoma from cellular adenomatous nodules and classic papillary thyroid carcinoma from papillary hyperplastic nodules can be challenging. Although immunohistochemistry is generally accepted as a useful ancillary technique in the diagnosis of papillary thyroid carcinoma, controversy exists regarding the best stain or combination of stains to distinguish papillary thyroid carcinoma from its mimics. Our study confirms the usefulness of HBME1 and suggests that the combination of HBME1 and CK19 attains a high sensitivity and specificity for the diagnosis of papillary thyroid carcinoma. Our results also demonstrate a lack of utility for CITED1 and SFTPB, which have recently been claimed to be sensitive markers for papillary thyroid carcinoma. Additionally, the two novel 
Table 2 Immunohistochemical expression of HBME1, FN1, CK19, CITED1, SFTPB, Calretinin, p16, Ki-67, CST6 and EPS8 in benign thyroid lesions

\begin{tabular}{|c|c|c|c|c|c|c|c|}
\hline \multirow[t]{2}{*}{ Immunostain } & \multicolumn{7}{|c|}{ Positive cases/total cases assessed (\%) } \\
\hline & Normal & $\begin{array}{l}\text { Adenomatous } \\
\text { nodule }\end{array}$ & $\begin{array}{l}\text { Graves } \\
\text { disease }\end{array}$ & $\begin{array}{c}\text { Papillary hyperplastic } \\
\text { nodule }\end{array}$ & $\begin{array}{l}\text { Follicular } \\
\text { adenoma }\end{array}$ & $\begin{array}{l}\text { Hashimoto's } \\
\text { thyroiditis }\end{array}$ & $\begin{array}{c}\text { Benign thyroids } \\
\text { (total) }\end{array}$ \\
\hline HBME1 & $0 / 10(0)$ & $0 / 10(0)$ & $0 / 8(0)$ & $0 / 4(0)$ & 0/6 (0) & 4/19 (21) & $4 / 57(7)$ \\
\hline CK19 & 10/10 (100) & $5 / 10(50)$ & 2/8 (25) & $1 / 4(25)$ & 5/6 (83) & 16/19 (84) & 39/57 (68) \\
\hline FN1 & 0/10 (0) & $1 / 10(10)$ & $0 / 8(0)$ & $0 / 4(0)$ & 2/6 (33) & $1 / 19(5)$ & $4 / 57$ (7) \\
\hline CITED1 & $10 / 10(100)$ & $1 / 1(100)$ & $2 / 3(67)$ & $1 / 1(100)$ & 6/6 (100) & $10 / 10(100)$ & $30 / 31$ (97) \\
\hline SFTPB & 0/10 (0) & 0/1 (0) & $0 / 3(0)$ & 0/1 (0) & $0 / 6(0)$ & $1 / 10(10)$ & $1 / 31(3)$ \\
\hline Ki-67 & $0 / 10(0)$ & $0 / 1(0)$ & $0 / 3(0)$ & $0 / 1(0)$ & $0 / 6(0)$ & 4/11 (36) & 4/32 (13) \\
\hline Calretinin & $0 / 5(0)$ & NA & NA & NA & NA & $0 / 5(0)$ & $0 / 10(0)$ \\
\hline p16 & $3 / 5(60)$ & NA & NA & NA & NA & $5 / 5(100)$ & 8/10 (80) \\
\hline CST6 & $6 / 8(75)$ & NA & NA & NA & NA & $5 / 7(71)$ & $11 / 15(73)$ \\
\hline EPS8 & $3 / 5(60)$ & NA & NA & NA & NA & $5 / 5(100)$ & 8/10 (80) \\
\hline
\end{tabular}

markers CST6 and EPS8 do not appear to be useful.

HBME1 is a monoclonal antibody directed against the microvillous surface of mesothelial cells. Although this antibody was originally developed as a mesothelioma marker, it was subsequently applied to the diagnosis of malignant thyroid conditions. ${ }^{22}$ Our findings of high sensitivity and specificity of HBME1 in the diagnosis of papillary thyroid carcinoma confirm previous studies that have found this antibody to be diagnostically useful., ${ }^{611,12,14}$ Although Miettinen's study showed HBME1 expression in all 145 papillary thyroid carcinomas, $33 \%$ of nodular goiters also showed some positivity. Of these, however, only five of $90(6 \%)$ showed staining in more than $10 \%$ of cells, a requirement for positivity in our study. Further, Miettinen and Karkkainen ${ }^{11}$ considered cytoplasmic and luminal staining without lateral membrane staining as positive whereas we considered such staining to be negative. Cheung et $a l^{6}$ reported HBME1 positivity in 38/54 (70\%) classic papillary thyroid carcinomas and 38/84 (45\%) follicular variant of papillary thyroid carcinomas with no expression in 40 nodular hyperplasia cases and 35 follicular adenomas. Similarly, Prasad et $a l^{13}$ demonstrated HBME1 expression in 57/67 $(85 \%)$ papillary thyroid carcinomas and only $1 / 102$ (1\%) non-neoplastic thyroid lesions stained for HBME1. The lower sensitivity of these and other studies compared to ours may be explained by their use of a lower titer of the HBME1 antibody. It should be remembered that HBME1 expression can be present in focal areas of Hashimoto's thyroiditis and the cells in these areas may show nuclear features of papillary thyroid carcinoma. HBME1 positivity per se should not be equated with a diagnosis of papillary thyroid carcinoma in this setting.

The diagnostic utility of CK19 in papillary thyroid carcinoma has been controversial. Some studies have reported negative CK19 staining in benign thyroid lesions and high frequencies of CK19 expression in papillary thyroid carcinoma. $^{23,24}$ Others, however, have observed focal positivity in benign thyroid lesions. ${ }^{10,25}$ Our study also noted a distressingly high rate of CK19 positivity in benign lesions. Not only was CK19 uniformly detected in all types of papillary thyroid carcinoma but it was also identified in $68 \%$ of benign thyroid lesions. Staining intensity was weak and diffuse in most of these benign lesions. These findings indicate that CK19 cannot be used by itself to establish a diagnosis of papillary thyroid carcinoma, although strong and diffuse CK19 expression should raise the suspicion of papillary thyroid carcinoma. The chief utility of CK19 lies in its high sensitivity for papillary thyroid carcinoma (100\% in our study). Negative staining for CK19, therefore, is strong evidence against papillary thyroid carcinoma. One situation where negative CK19 staining is especially helpful is in papillary thyroid hyperplasia, which can simulate papillary thyroid carcinoma morphologically. In our study, 3/4 papillary hyperplastic nodules were negative for CK19 whereas focal staining $(15 \%)$ was present in one.

Fibronectin1 has been proposed as a useful marker to diagnose papillary thyroid carcinoma. ${ }^{13,14,26}$ Although FN1 was fairly specific in our study, a large proportion of papillary thyroid carcinomas $(31 \%)$ were negative. Even those cases that were positive showed weak and focal staining, and there was often significant background staining. The focal nature of FN1 staining may explain why small papillary thyroid carcinomas (microcarcinomas) had such a low rate of FN1 positivity in our series. In our experience, low sensitivity, focal staining and high background positivity significantly impair the utility of FN1 in a diagnostic setting.

CITED1 and SFTPB have recently been claimed to be sensitive markers for papillary thyroid carcinoma. ${ }^{15,16}$ Huang et $a l^{15}$ showed that CITED1 and SFTPB expression was detected in $49 / 52(94 \%)$ and 

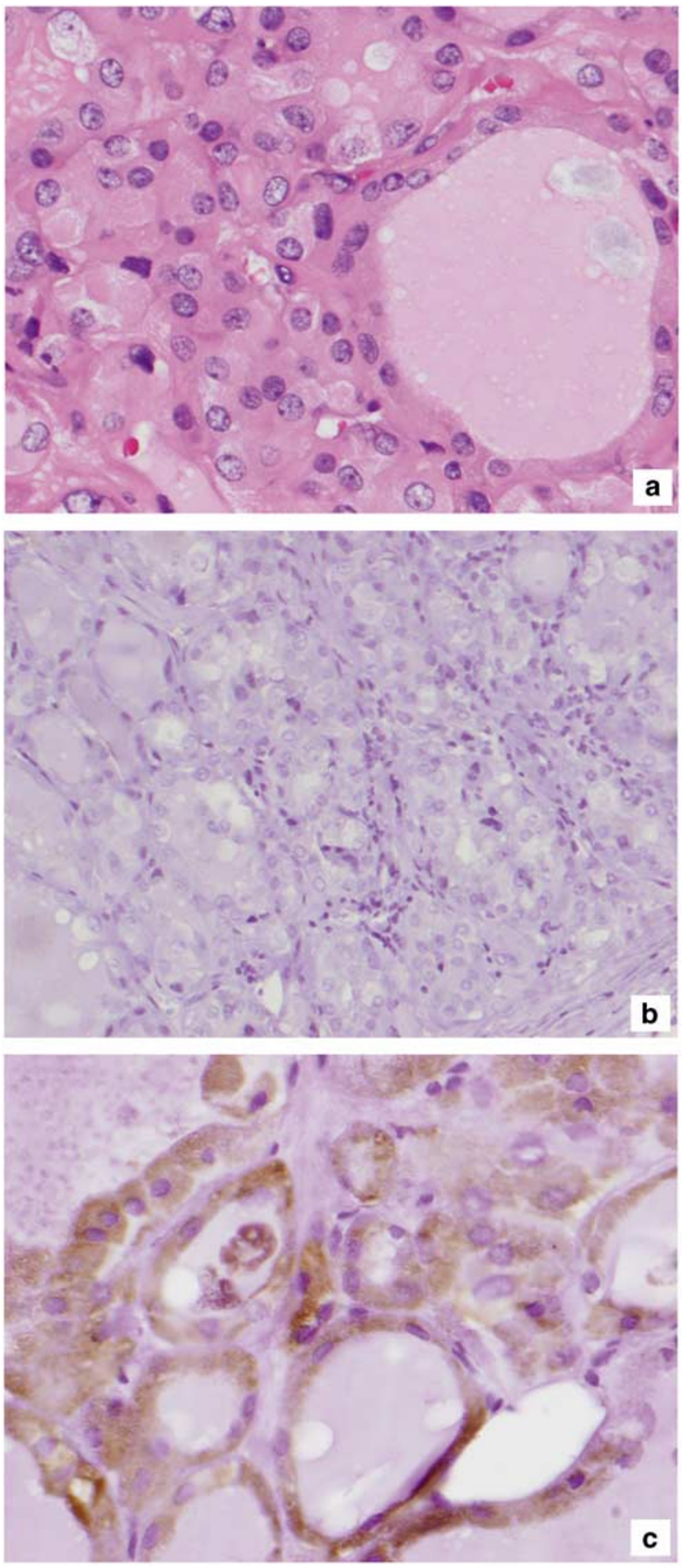

Figure 4 Adenomatous nodule. (a) H\&E $(\times 100)$. The nodule is negative for HBME1 (b) but positive for CK19 (c) (b and $\mathbf{c}, \times 100)$.

39/52 (75\%) papillary thyroid carcinomas, respectively, but not in follicular carcinoma and normal thyroid tissue. Similarly, Prasad et $a l^{16}$ demonstrated that CITED1 is preferentially expressed in papillary thyroid carcinoma and suggested that it could be of diagnostic value. In contrast to these

\section{References}

1 Greenlee RT, Hill-Harmon MB, Murray T, et al. Cancer statistics, 2001. CA Cancer J Clin 2001;51:15-36.

2 Finley DJ, Arora N, Zhu B, et al. Molecular profiling distinguishes papillary carcinoma from benign thyroid nodules. J Clin Endocrinol Metab 2004;89: 3214-3223.

3 Rosai J, Zampi G, Carcangiu ML. Papillary carcinoma of the thyroid: a discussion of its several morphologic expressions, with particular emphasis on the follicular variant. Am J Surg Pathol 1983;7:809-817.

4 Chem KT, Rosai J. Follicular variant of thyroid papillary carcinoma: a clinicopathological study of six cases. Am J Surg Pathol 1977;1:123-130.

5 Rosai J, Carcangiu ML, DeLellis RA. Tumors of the thyroid gland. In: Rosai J, Carcangiu ML, DeLellis RA (eds). Atlas of Tumor Pathology, Third Series, Fascicle 5. Armed Forces Institute of Pathology: Washington, DC, 1992, pp 65-121.

6 Cheung CC, Ezzat S, Freeman JL, et al. Immunohistochemical diagnosis of papillary thyroid carcinoma. Mod Pathol 2001;14:338-342. 
7 Raphael SJ, McKeown-Eyssen G, Asa SL. High-molecular-weight cytokeratin and cytokeratin-19 in the diagnosis of thyroid tumors. Mod Pathol 1994;7: 295-300.

8 Fonseca E, Nesland JM, Hoie J, et al. Pattern of expression of intermediate cytokeratin filaments in the thyroid gland: an immunohistochemical study of simple and stratified epithelial-type cytokeratins. Virchows Arch 1997;430:239-345.

9 Baloch ZW, Abraham S, Roberts S, et al. Differential expression of cytokeratins in follicular variant of papillary carcinoma: an immunohistochemical study and its diagnostic utility. Hum Pathol 1999;30: 1166-1171.

10 Casey MB, Lohse CM, Lloyd RV. Distinction between papillary thyroid hyperplasia and papillary thyroid carcinoma by immunohistochemical staining for cytokeratin 19, galectin-3, and HBME-1. Endocr Pathol 2003;14:55-60.

11 Miettinen M, Karkkainen P. Differential reactivity of HBME-1 and CD15 antibodies in benign and malignant thyroid tumours, Preferential reactivity with malignant tumours. Virchows Arch 1996;429:213-219.

12 Sack MJ, Astengo-Osuna C, Lin BT, et al. HBME-1 immunostaining in thyroid fine-needle aspirations: a useful marker in the diagnosis of carcinoma. Mod Pathol 1997;10:668-674.

13 Prasad ML, Pellegata NS, Huang Y, et al. Galectin-3, fibronectin-1, CITED1, HBME1 and cytokeratin-19 immunohistochemistry is useful for the differential diagnosis of thyroid tumors. Mod Pathol 2005; 18:48-57.

14 Prasad ML, Huang Y, Pellegata NS, et al. Hashimoto's thyroiditis with papillary thyroid carcinoma (PTC)like nuclear alterations express molecular markers of PTC. Histopathology 2004;45:39-46.

15 Huang Y, Prasad M, Lemon WJ, et al. Gene expression in papillary thyroid carcinoma reveals highly consistent profiles. Proc Natl Acad Sci USA 2001;98: 15044-15049.

16 Prasad ML, Pellegata NS, Kloos RT, et al. CITED1 protein expression suggests papillary thyroid carci- noma in high throughput tissue microarray-based study. Thyroid 2004;14:169-175.

17 Xu XC, el-Naggar AK, Lotan R. Differential expression of galectin-1 and galectin-3 in thyroid tumors. Potential diagnostic implications. Am J Pathol 1995;147: 815-822.

18 Inohara $\mathrm{H}$, Honjo $\mathrm{Y}$, Yoshii $\mathrm{T}$, et al. Expression of galectin-3 in fine-needle aspirates as a diagnostic marker differentiating benign from malignant thyroid neoplasms. Cancer 1999;85:2475-2484.

19 Bartolazzi A, Bussolati G. Galectin-3 does not reliably distinguish benign from malignant thyroid neoplasms. Histopathology 2006;48:212-213.

20 Mills LJ, Poller DN, Yiangou C. Galectin-3 is not useful in thyroid FNA. Cytopathology 2005;16: 132-138.

21 Mehrotra P, Okpokam A, Bouhaidar R, et al. Galectin-3 does not reliably distinguish benign from malignant thyroid neoplasms. Histopathology 2004;45:493-500.

22 Sheibani K, Esteban JM, Bailey A, et al. Immunopathologic and molecular studies as an aid to the diagnosis of malignant mesothelioma. Hum Pathol 1992;23: 107-116.

23 de Matos PS, Ferreira AP, de Oliveira Facuri F, et al. Usefulness of HBME-1, cytokeratin 19 and galectin-3 immunostaining in the diagnosis of thyroid malignancy. Histopathology 2005;47:391-401.

24 Khurana KK, Truong LD, LiVolsi VA, et al. Cytokeratin 19 immunolocalization in cell block preparation of thyroid aspirates. An adjunct to fine-needle aspiration diagnosis of papillary thyroid carcinoma. Arch Pathol Lab Med 2003;127:579-583.

25 Cameron BR, Berean KW. Cytokeratin subtypes in thyroid tumours: immunohistochemical study with emphasis on the follicular variant of papillary carcinoma. J Otolaryngol 2003;32:319-322.

26 Takano T, Miyauchi A, Yokozawa T, et al. Preoperative diagnosis of thyroid papillary and anaplastic carcinomas by real-time quantitative reverse transcription-polymerase chain reaction of oncofetal fibronectin messenger RNA. Cancer Res 1999;59: 4542-4545. 